Check for updates

Cite this: RSC Adv., 2019, 9, 37512

\title{
In situ synthesis of monolayer graphene on silicon for near-infrared photodetectors
}

\author{
Pengcheng Xiang, ${ }^{a}$ Gang Wang, (D) *a Siwei Yang, ${ }^{\mathrm{b}}$ Zhiduo Liu, (D) ce Li Zheng, (D) b \\ Jiurong Li, ${ }^{a}$ Anli Xu, ${ }^{\text {be }}$ Menghan Zhao, ${ }^{a}$ Wei Zhu, ${ }^{a}$ Qinglei Guo*df and Da Chen ${ }^{\star a}$
}

Direct integration of monolayer graphene on a silicon (Si) substrate is realized by a simple thermal annealing process, involving a top copper (Cu) layer as the catalyst and an inserted polymethylmethacrylate (PMMA) as the carbon source. After spin-coating the PMMA carbon source on the Si substrate, the Cu catalyst was deposited on PMMA/Si by electron beam evaporation. After that, graphene was directly synthesized on $\mathrm{Si}$ by decomposition and dehydrogenation of PMMA and the catalyzation effect of $\mathrm{Cu}$ under a simple thermal annealing process. Furthermore, under an optimized growth condition, monolayer graphene directly formed on the $\mathrm{Si}$ substrate was demonstrated. Utilizing the as-grown graphene/Si heterojunction, near-infrared photodetectors with high detectivity $\left(\sim 1.1 \times 10^{10} \mathrm{~cm} \mathrm{~Hz} z^{1 / 2} W^{-1}\right)$ and high responsivity $\left(50 \mathrm{~mA} \mathrm{~W}^{-1}\right)$ at $1550 \mathrm{~nm}$ were directly fabricated without any post-transfer process. The proposed approach for directly growing graphene on silicon is highly scalable and compatible with present nano/micro-fabrication systems, thus promoting the application of graphene in microelectronic fields.

Received 28th August 2019 Accepted 11th November 2019

DOI: $10.1039 / c 9 r a 06792 b$

rsc.li/rsc-advances and $\mathrm{Al}_{2} \mathrm{O}_{3}$, has been recently demonstrated. ${ }^{8-12}$ However, singlecrystalline $\mathrm{Si}$, the most common commercial semiconductor, attracted scant attention when utilized as the substrate for direct synthesis of graphene. Previous works showed that the pre-formation of Si carbide during the PMMA decomposition process weakens the nucleation and growth of graphene. ${ }^{\mathbf{1 3 , 1 4}}$ Although solid source molecular-beam epitaxy or CVD without a metallic catalyst may also provide possibilities, only graphitic films have been accomplished. ${ }^{15-17}$

In this work, we report a practical and straightforward technology to directly synthesize graphene on Si substrates. The key idea of this approach is to form a uniform PMMA on Si, followed by depositing a $\mathrm{Cu}$ layer on the PMMA as the catalyst. Notably, the PMMA appears to have a dual-role: (i) as the carbon source to synthesize graphene film; (ii) as the barrier to prevent $\mathrm{Cu}$ diffusing into Si substrate. We should stress that $\mathrm{Cu}$ atom residuals are still found in silicon after graphene synthesis although the fraction is meager. According to the optimized growth condition, the highest annealing temperature should not exceed $900{ }^{\circ} \mathrm{C}$, aiming to prevent the wetting and evaporation of $\mathrm{Cu}$ layer and the annealing time is shortened to be 13 min. Finally, high-performance near-infrared photodetectors are directly fabricated by utilizing the as-grown graphene/Si heterojunctions. Electrical measurement results show that the as-grown graphene/Si heterojunction has a typical current rectification behavior, indicating a Schottky barrier between graphene and Si. Moreover, the graphene/Si based near-infrared photodetector with outstanding photovoltaic conversion capability exhibits high-performances in responsivity $\left(50 \mathrm{~mA} \mathrm{~W}^{-1}\right)$ 
and detectivity $\left(\sim 1.1 \times 10^{10} \mathrm{~cm} \mathrm{~Hz}^{1 / 2} \mathrm{~W}^{-1}\right)$ when illuminated with a $1550 \mathrm{~nm}$ light.

\section{Materials and methods}

Fig. 1a displays the schematic process flow for directly synthesizing graphene on Si. After cleaning the Si substrate by Radio Corporation of American (RCA), the native oxide layer was removed by buffered oxide etch (BOE). A $200 \mu \mathrm{L}$ PMMA (MicroChem Corp. 950, A4) in toluene with a concentration of $0.025 \mathrm{wt} \%$ yielded about $2 \mathrm{~nm}$-thick PMMA film at a spin-coat rate of 8000 for $100 \mathrm{~s}$. The thicknesses of PMMA film was determined by ellipsometry. Then a $30 \mathrm{~nm}$-thick $\mathrm{Cu}$ layer was deposited on the Si/PMMA as the catalyst. Subsequently, the Si/ PMMA/Cu sample was loaded into a CVD chamber for thermal annealing under a $\mathrm{H}_{2} / \mathrm{Ar}$ flow, and graphene was formed and sandwiched by $\mathrm{Si}$ substrate and $\mathrm{Cu}$ capping layer. After the removal of $\mathrm{Cu}$ layer by chemical etching process in $20 \%$ $\left(\mathrm{NH}_{4}\right)_{2} \mathrm{~S}_{2} \mathrm{O}_{8}$ aqueous solution, graphene is exposed on the $\mathrm{Si}$ substrate.

The growth condition for high-quality graphene is optimized by tuning the annealing temperature, growth time, and gas atmosphere. For materials characterizations, the layer number, quality, and uniformity of the as-grown graphene were evaluated by Raman scattering (HORIBA Jobin Yvon HR800), atomic force microscopy (AFM, Multimode 8) measurement and transmission electron microscope (TEM, FET-Tecnai G2F20 S7WIN); the element distribution along the depth of the asgrown graphene was analyzed by secondary ion mass spectrometry (SIMS, Cameca IMS-4F, Paris, France); the surface chemical composition of as-grown graphene was revealed by $\mathrm{X}$ ray photoelectron spectroscopy (XPS, PHI 5802, Physical Electronics Inc, Eden Prairie, MN); the crystalline quality and microstructure of the as-grown graphene were accessed by scanning tunneling microscope (STM, SPECS JT-STM) in the constant current mode; electrical properties of graphene/Si based devices were investigated via a semiconductor parameter analyzer (B1500A and Keithley 4200).

\section{Results and discussion}

\subsection{Condition optimization for graphene preparation}

Fig. 1b indicates the Raman results of the graphene grown at different temperatures of $810,840,870$ and $900{ }^{\circ} \mathrm{C}$. At $810{ }^{\circ} \mathrm{C}$ or lower, three representative peaks locating at $1350 \mathrm{~cm}^{-1}$, $1580 \mathrm{~cm}^{-1}$, and $2700 \mathrm{~cm}^{-1}$ are found, which are known as the characteristic peaks of graphene, ${ }^{\mathbf{8} 9}$ i.e., D-peak, G-peak and 2Dpeak, respectively. The calculated peak intensity ratio, $I_{\mathrm{D}} / I_{\mathrm{G}}$, of graphene synthesized at a temperature of $810^{\circ} \mathrm{C}$ is about 1 , implying the presence of defects. ${ }^{18}$ As the growth temperature increases, the intensity of D-peak gradually attenuates, suggesting an improved crystalline quality. Notably, no appreciable D-peak is observed at $900{ }^{\circ} \mathrm{C}$. Therefore, the optimized temperature for graphene growth is $900{ }^{\circ} \mathrm{C}$. In addition to the annealing temperature, the annealing time is also regarded as an important factor for obtaining graphene with high-quality. ${ }^{\mathbf{1 9}}$ With an annealing time of $4 \mathrm{~min}$, a distinct D-band is observed, as shown in Fig. 1c. As the annealing time increasing, the Dpeak is suppressed to disappear when annealed for $13 \mathrm{~min}$. Prolonging the annealing time, such as 15 min or longer, the obtained Raman spectra (not shown here) are similar to the sample annealed for $13 \mathrm{~min}$. Previous works demonstrated that PMMA starts to decompose even the annealing temperature is low $\left(\sim 600{ }^{\circ} \mathrm{C}\right),{ }^{14}$ therefore, complete PMMA decomposition and graphene formation should need sufficient annealing temperature and time. Once the annealing time is over $13 \mathrm{~min}$, the graphene quality will not be further improved. The growth condition was further optimized by changing the $\mathrm{Ar}: \mathrm{H}_{2}$ flow rate ratio (sccm), as shown in Fig. 1d. The annealing temperature and time are fixed at $900{ }^{\circ} \mathrm{C}$ and $13 \mathrm{~min}$. As the $\mathrm{Ar}: \mathrm{H}_{2}$ flow rate ratio is increased from $50: 10$ to $50: 1$, the intensity of $\mathrm{D}$ peak for graphene decreases rapidly, indicating an improved
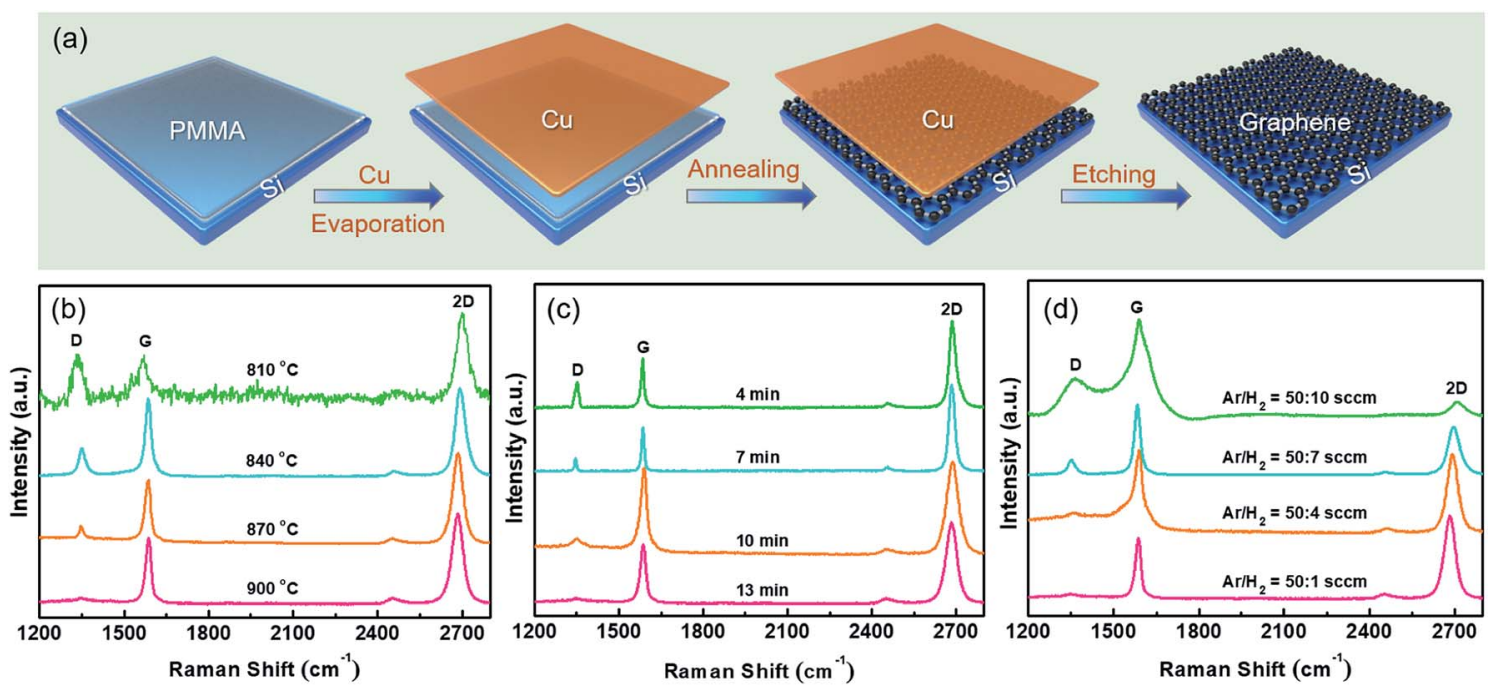

Fig. 1 Condition optimization for graphene preparation. (a) Schematic process flow of synthesizing graphene on Si. (b-d) Raman results of the as-grown graphene on $\mathrm{Si}$ at various grown conditions: (b) temperatures, (c) annealing times, and (d) $\mathrm{Ar} / \mathrm{H}_{2} \mathrm{ratios}$. 
crystalline quality. When the $\mathrm{H}_{2}$ flow is reduced to $1 \mathrm{sccm}$, the D-band completely disappears.

\subsection{The preparation of graphene on $\mathrm{Si}$ and its characterization}

In our experiment, water-solvable PMMA was selected because of its capability of forming large-area uniform film with tunable thickness on various substrates by spin-coating. ${ }^{20}$ A conformal PMMA coating layer on the desired substrate has been demonstrated as a vital step for synthesizing high-quality graphene, which can be attributed to the intimate relationship between the layer number of graphene and the carbon distribution in PMMA. ${ }^{21,22}$ In order to verify the uniformity of PMMA spin-coated on Si, AFM measurement was performed. As shown in Fig. 2a, the surface roughness of PMMA is about $1.1 \mathrm{~nm}$ over a scanned area of $45 \times 45 \mu^{2}$. The surface roughness has no significant change after exposing graphene which is formed under the above optimized condition (temperature: $900{ }^{\circ} \mathrm{C}$; time: $13 \mathrm{~min} ; \mathrm{H}_{2}$ to Ar flow rate ratio: $50: 1$ ), as demonstrated in Fig. $2 \mathrm{~b}$ with a value of about $1.2 \mathrm{~nm}$. The residual $\mathrm{Cu}$ atoms in graphene/Si were investigated by secondary ion mass spectrometry (SIMS). As displayed in Fig. 2c, the fraction of $\mathrm{Cu}$ atoms residual in the $\mathrm{Si}$ substrate is extremely low with a concentration even below the detection limit of the instruments. We also notice that carbon atoms are also found in the $\mathrm{Si}$ substrate although the fraction is rather low.

Fig. 2d displays the Raman spectrum of the as-grown graphene synthesized at the optimized condition. The strongly suppressed D-peak indicates that the as-grown graphene has a comparable quality to the graphene grown by conventional
CVD. ${ }^{9}$ From the Lorentzian fitting curve of 2D-peak (see inset of Fig. 2d), the corresponding full-width at half-maximum (FWHM) is extracted as about $35 \mathrm{~cm}^{-1}$, evidencing the optimized graphene is monolayer. ${ }^{18}$ From the XPS analysis of the asgrown sample shown in Fig. 2e, Si, carbon (C) and oxygen (O) characteristic peaks are found. A $\mathrm{sp}^{2}$-hybridization peak of carbon, locating at $\sim 284.3 \mathrm{eV}$, is exposed from the deconvolution of C-1s spectrum (inset, Fig. 2e). Therefore, one can conclude that the $\mathrm{sp}^{2}$ network, i.e., graphene, is formed. ${ }^{23}$ The minor peak locating at $\sim 285.3 \mathrm{eV}$ denotes the $\mathrm{sp}^{3}$-hybridization is generated from the natural carbon contamination. ${ }^{24}$ Fig. $2 f$ shows the STM measurement of the as-grown graphene, and the magnified high-resolution STM result, with a representative honeycomb lattice structure, ${ }^{\mathbf{1 8}}$ is displayed in the inset. No visible lattice defect is observed within the area greater than $8 \mathrm{~nm} \times 8 \mathrm{~nm}$, indicating the high crystalline quality of the assynthesized graphene.

\subsection{Thickness and electrical property evaluation of the synthesized graphene}

Fig. 3a shows the TEM (left panel) image of the synthesized graphene, and a continuous graphene over a large area is observed. From the selected area electron diffraction (SAED, left inset) result that one set of hexagonal diffraction pattern is presented, the as-grown graphene has a single-crystalline lattice structure. The layer number of the as-grown can be intuitively confirmed by the high-resolution TEM (HR-TEM) result, as shown in the right of Fig. 3a. Only one straight line is observed, indicating the as-grown graphene is monolayer. In our work, direct synthesis of monolayer graphene on arbitrary substrate
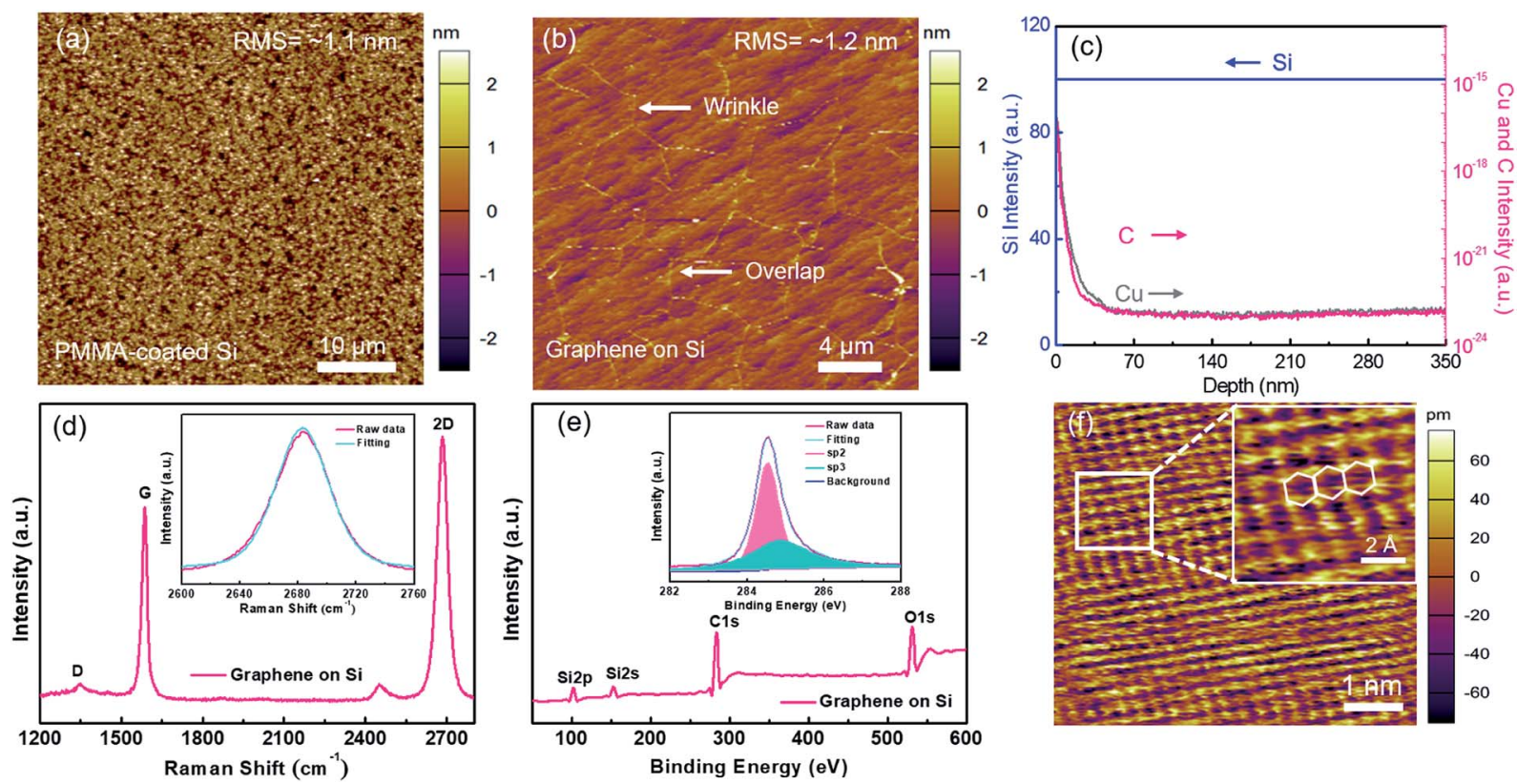

Fig. 2 The preparation of graphene on Si and its characterization. Surface morphology and roughness analyses of: (a) PMMA-coated Si substrate and (b) as-grown graphene on Si substrate. (c) SIMS depth profiles of Cu atoms in Si substrate. Cu/PMMA/Si system after graphene growth and Cu layer removal. (d) Raman spectrum of as-grown graphene on Si substrate under the optimized condition; Lorentzian fitting of the 2D band is performed to determine the FWHM (inset). (e) XPS analysis of graphene/Si after removing Cu. (f) STM image of as-grown graphene on Si. 

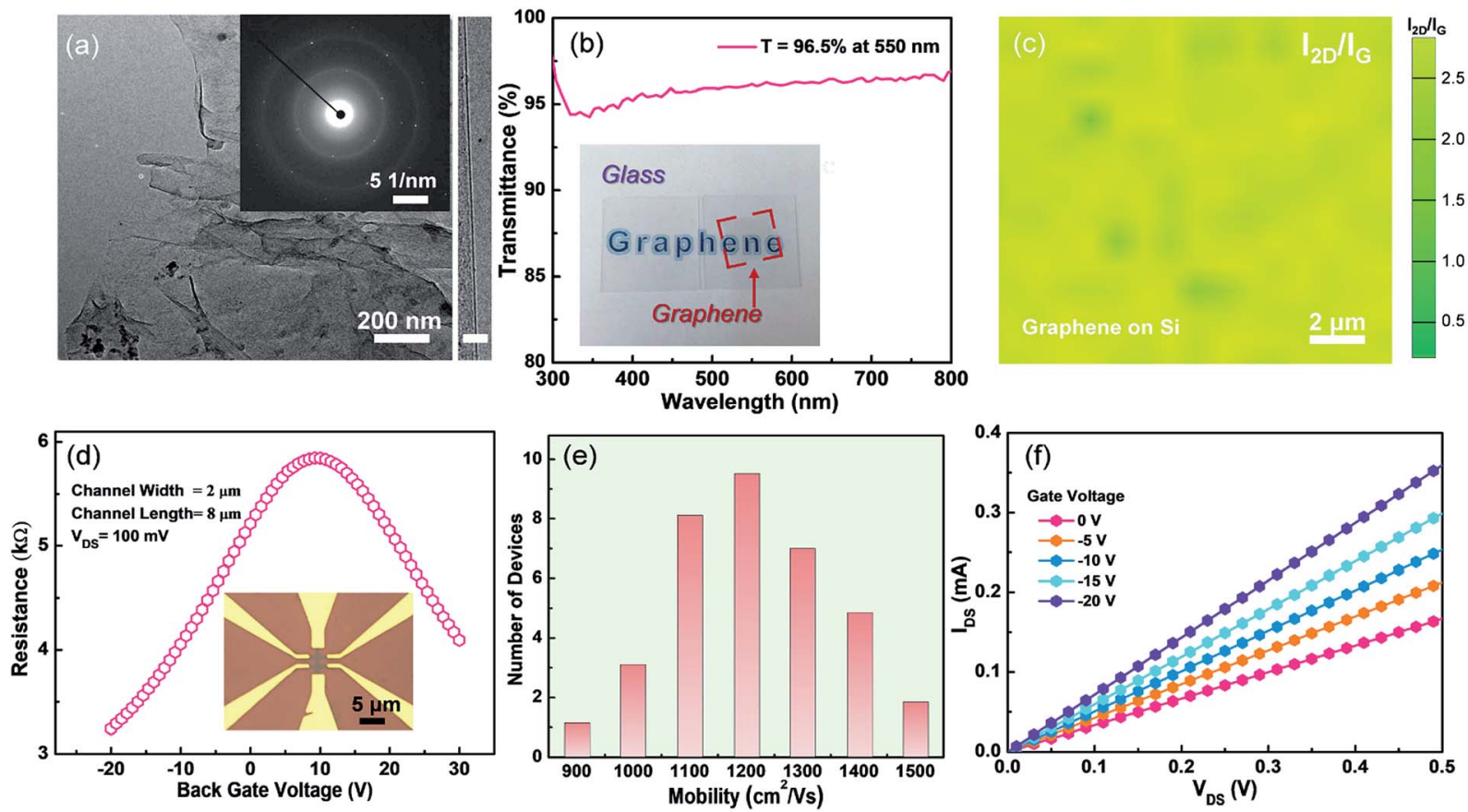

Fig. 3 Thickness and electrical property evaluation of the as-synthesized graphene. (a) TEM (left; inset: SAED pattern) and HR-TEM (right) images of the as-grown graphene on Si substrate. Scale bar is $3 \mathrm{~nm}$. (b) Optical transmittance of the directly grown monolayer graphene on glass substrate. A digital image of graphene on $1 \times 1 \mathrm{~cm}^{2}$ quartz is displayed in the inset. (c) Raman mapping results of $I_{2 D} / I_{G}$ measured from the asgrown graphene. (d) $I_{\mathrm{DS}}-V_{\mathrm{G}}$ curves of back-gated GFET with directly grown monolayer graphene on $\mathrm{SiO}_{2} / \mathrm{Si}$ substrate as channel, $V_{\mathrm{DS}}=100 \mathrm{mV}$. A typical device image is shown in the inset. (e) Histogram of the mobility distribution acquired from 36 GFETs. (f) Output (/DS $\left.-V_{D S}\right)$ characteristics of the GFET at different $V_{G}$.

(such as $\mathrm{SiO}_{2}$ and glass) by utilizing copper-assisted chemical vapor deposition, without any post-transfer process for optical transmittance and transport properties measurements. To further investigate the layer number, the optical transmittance of as-grown graphene on glass is characterized and the result is presented in Fig. 3b. The transmittance is $96.5 \%$ at the wavelength of $550 \mathrm{~nm}$. Because the absorbance for an individual graphene layer is about $2.3 \%,{ }^{25}$ the layer number of as-grown graphene can be estimated to be only one atom layer, which is consistent with HR-TEM and Raman results. Moreover, the inset of Fig. 3b shows that monolayer graphene has good transparency and macroscopic uniformity. To determine the quality and uniformity, a random area $30 \mu \mathrm{m} \times 30 \mu \mathrm{m}$ of the asgrown graphene is selected for Raman mapping measurement. Fig. 3c shows the extracted ratios of $2 \mathrm{D}$ to G peak intensity. The $I_{2 \mathrm{D}} / I_{\mathrm{G}}$ is in a range of 2-2.5, conforming the graphene grown on Si substrate by the presented method is homogenous and uniform.

Good transport properties, especially the carrier mobility, of graphene determines its potentials for the applications in highperformance electronic devices. In order to investigate the electrical transport ability of the graphene grown by our proposed method, graphene-based field effect transistors (GFETs) with a back gate were fabricated and characterized. Fig. 3d shows a typical resistance-gate voltage curve measured from the GFET. After measuring 36 GFETs, the calculated hole mobility mainly locates at $1000-1200 \mathrm{~cm}^{2} \mathrm{~V}^{-1} \mathrm{~s}^{-1}$, and the electron mobility is in the range of $1200-1400 \mathrm{~cm}^{2} \mathrm{~V}^{-1} \mathrm{~s}^{-1}$, are shown in Fig. 3e. We should stress that these carrier mobility values for hole and electron are comparable to recently reported values measured from the CVD graphene. ${ }^{9,18,26} \mathrm{Fig}$. $3 \mathrm{f}$ shows the output characteristics of GFETs. Under arbitrary biased gate voltage, $I_{\mathrm{DS}}$ always increases linearly with $V_{\mathrm{DS}}$. Therefore, the contact type between electrode ( $\mathrm{Ti} / \mathrm{Au})$ and graphene is ohmic. Notably, $I_{\mathrm{DS}}$ decreases monotonously with the increase of $V_{\mathrm{G}}$ swapping from -20 to $0 \mathrm{~V}$, implying a slight p-doping in the assynthesized graphene. ${ }^{27}$

\subsection{Responsivity and detectivity of graphene/Si based photodetector}

Photodetectors have exhibited enormous potentials in optical communications, biomedical imaging, and motion detection. ${ }^{28}$ In terms of high-performance optoelectronic devices, graphene has attracted significant attentions due to its superior electronic and optical properties. For our proposed method, the significant advantage is directly integrating the as-grown graphene/Si heterojunction into a Schottky junction-based photodetector, because the average Schottky barriers of graphene/silicon junctions are estimated to be $0.45-0.47 \mathrm{eV} .^{10}$ The performances of graphene/Si photodetectors are assessed by measuring the current passing through the Schottky heterojunction at dark and light illumination conditions, as shown in Fig. 4a. Without the illumination, typical rectifying behavior is observed, further confirming the contact between graphene and 

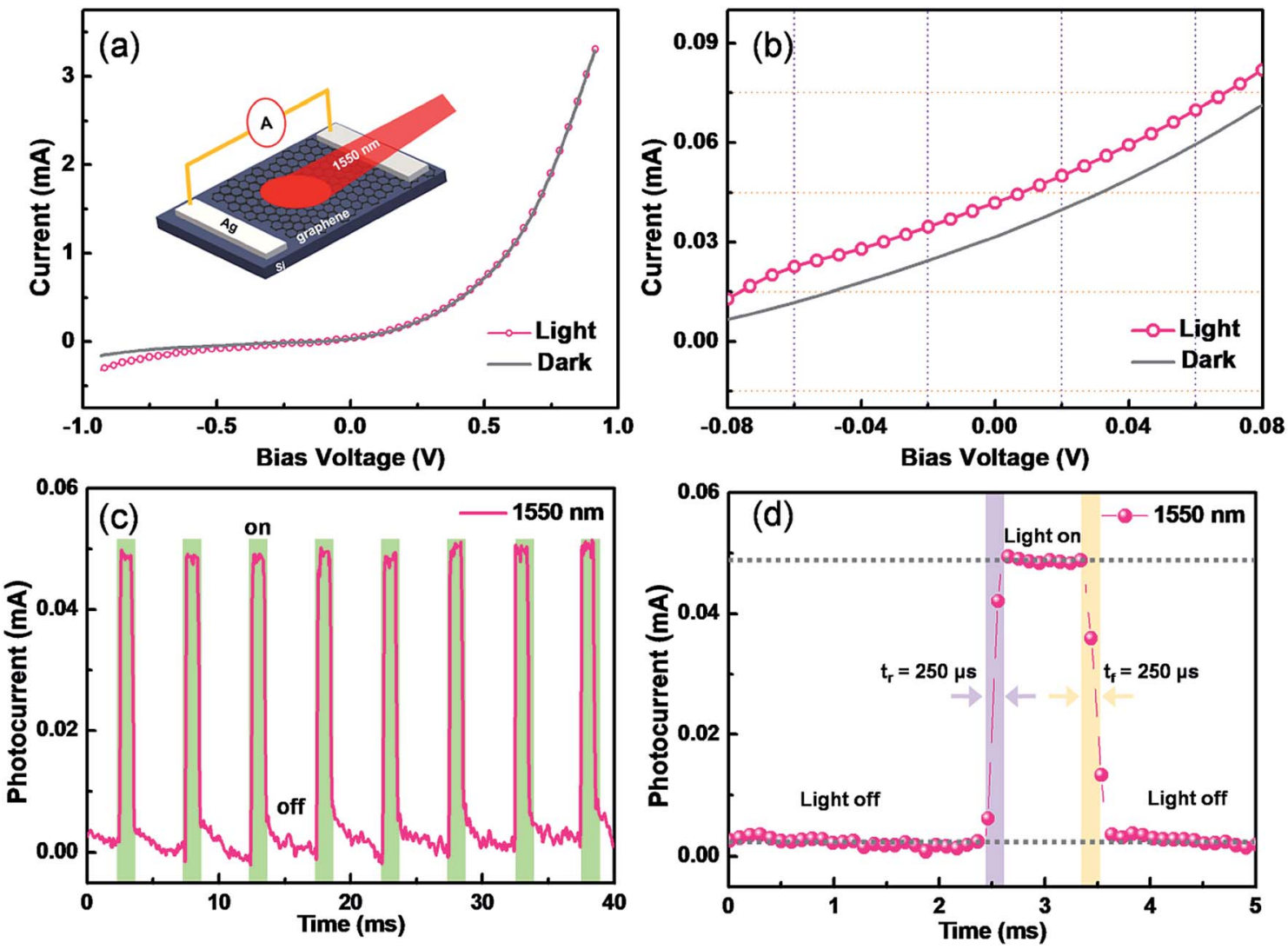

Fig. 4 Responsivity and detectivity of graphene/Si based photodetector. (a) Currents of the graphene/Si based photodetector as a function of bias voltage with/without light illumination. A schematic illustration of the photodetector is shown in the inset. (b) The magnified $I-V$ characteristic in a low voltage range. (c) Photocurrent of the photodetector illuminated with a pulsed $1550 \mathrm{~nm}$ light, and the power density is about 27 $\mathrm{mW} \mathrm{cm} \mathrm{cm}^{-2}$. (d) Enlarged photovoltage of the graphene/Si Schottky photodetector responded to a pulsed illumination.

Si is Schottky-type. ${ }^{29,30}$ When illuminated by a $1550 \mathrm{~nm}$ light, the reversed current is significantly enhanced. ${ }^{31}$ To investigate more details, a magnification of the $I-V$ characteristic is processed and shown in Fig. 4b, and a typical photovoltaic effect is found. ${ }^{10}$ Fig. $4 \mathrm{c}$ depicts the photocurrent of a graphene/Si photodetector varying with the illumination from a pulsed 1550 light. The bias voltage is set as zero. Highly reversible lowor high-resistivity states are demonstrated. Fig. 4d shows the enlarged transient photo-response curve, where both rise time $\left(t_{\mathrm{r}}\right)$ and fall time $\left(t_{\mathrm{f}}\right)$ are estimated to be only $250 \mu \mathrm{s}$. Moreover, the detectivity and responsivity are calculated to be as high as $\sim 1.1 \times 10^{10} \mathrm{~cm} \mathrm{~Hz}^{1 / 2} \mathrm{~W}^{-1}$ and $\sim 50 \mathrm{~mA} \mathrm{~W} \mathrm{~W}^{-1}$, respectively, suggesting great potentials in logic and photoelectric circuits.

\section{Conclusions}

In summary, we describe a simple and convenient approach to directly synthesize graphene on single-crystalline silicon substrate. The quality of synthesized graphene is optimized via tuning the growth conditions. To investigate the optoelectronic properties, Schottky junction based photodetectors are directly constructed by utilizing the as-grown graphene/Si. Optoelectronic results indicate that the as-grown graphene/Si based photodetector exhibits a distinct photovoltaic behavior, with high responsivity and detectivity of $\sim 50 \mathrm{~mA} \mathrm{~W}{ }^{-1}$ and $\sim 1.1 \times$ $10^{10} \mathrm{~cm} \mathrm{~Hz}^{1 / 2} \mathrm{~W}^{-1}$, respectively. Our study may pave the way for synthesizing graphene on arbitrary substrate in a highly scalable way, thus promoting applications of graphene in microelectronic and optoelectronic fields.

\section{Conflicts of interest}

The authors declare that they have no conflict of interest.

\section{Acknowledgements}

The authors thank the financial support from National Natural Science Foundation of China under Grant (No. 11704204, 11804353, 61604084, 51602056 and 11774368), Scientific Research Fund Project in Ningbo University under Grant (XYL18024), K. C. Wong Magna Fund in Ningbo University and the Natural Science Foundation of Ningbo under Grant (No. 2017A610104).

\section{References}

1 A. K. Geim, Science, 2009, 324, 1530-1534.

2 M. Yi and Z. G. Shen, J. Mater. Chem. A, 2015, 3, 11700-11715. 
3 X. S. Li, L. Colombo and R. S. Ruoff, Adv. Mater., 2016, 28, 6247-6252.

4 X. F. Zhang, T. R. Wu, Q. Jiang, H. S. Wang, H. L. Zhu, Z. Y. Chen, R. Jiang, T. C. Niu, Z. J. Li, Y. W. Zhang, Z. J. Qiu, G. H. Yu, A. Li, S. Qiao, H. M. Wang, Q. K. Yu and X. M. Xie, Small, 2019, 15, 1805395.

5 Q. K. Yu, L. A. Jauregui, W. Wu, R. Colby, J. F. Tian, Z. H. Su, H. L. Cao, Z. H. Liu, D. Pandey, D. G. Wei, T. F. Chung, P. Peng, N. P. Guisinger, E. A. Stach, J. M. Bao, S. S. Pei and Y. P. Chen, Nat. Mater., 2011, 10, 443-449.

6 S. Kasap, H. Khaksaran, S. Celik, H. Ozkaya, C. Yanık and I. I. Kaya, Phys. Chem. Chem. Phys., 2015, 17, 23081-23087.

7 K. S. Novoselov, V. I. Fal'ko, L. Colombo, P. R. Gellert, M. G. Schwab and K. Kim, Nature, 2012, 490, 192-200.

8 J. H. Lee, E. K. Lee, W. J. Joo, Y. Jang, B. S. Kim, J. Y. Lim, S. H. Choi, S. J. Ahn, J. R. Ahn, M. H. Park, C. W. Yang, B. L. Choi, S. W. Hwang and D. Whang, Science, 2014, 344, 286-289.

9 G. Wang, M. Zhang, Y. Zhu, G. Q. Ding, D. Jiang, Q. L. Guo, S. Liu, X. M. Xie, P. K. Chu, Z. F. Di and X. Wang, Sci. Rep., 2013, 3, 2465.

10 J. R. Li, Q. L. Guo, N. Zhang, S. W. Yang, Z. D. Liu, A. L. Xu, W. D. Tao, G. Wang, D. Chen and G. Q. Ding, J. Mater. Chem. C, 2018, 6, 9682-9690.

11 Z. W. Peng, Z. Yan, Z. Z. Sun and J. M. Tour, ACS Nano, 2011, 5, 8241-8247.

12 W. Yang, G. R. Chen, Z. W. Shi, C. C. Liu, L. C. Zhang, G. B. Xie, M. Cheng, D. M. Wang, R. Yang, D. X. Shi, K. J. Watanabe, T. Taniguchi, Y. G. Yao, Y. B. Zhang and G. Y. Zhang, Nat. Mater., 2013, 12, 792-797.

13 Z. Z. Sun, Z. Yan, J. Yao, E. Beitler, Y. Zhu and J. M. Tour, Nature, 2010, 468, 549-552.

14 J. Hofrichter, B. N. Szafranek, M. Otto, T. J. Echtermeyer, M. Baus, A. Majerus, V. Geringer, M. Ramsteiner and H. Kurz, Nano Lett., 2010, 10, 36-42.

15 W. Strupinski, K. Grodecki, A. Wysmolek, R. Stepniewski, T. Szkopek, P. E. Gaskell, A. Grüneis, D. Haberer, R. Bozek, J. Krupka and J. M. Baranowski, Nano Lett., 2011, 11, 17861791.

16 J. Kwak, T. Y. Kwon, J. H. Chu, J. K. Choi, M. S. Lee, S. Y. Kim, H. J. Shin, K. Park, J. U. Park and S. Y. Kwon, Phys. Chem. Chem. Phys., 2013, 15, 10446-10452.
17 S. K. Jerng, D. S. Yu, Y. S. Kim, J. Ryou, S. Hong, C. Kim, S. Yoon, D. K. Efetov, P. Kim and S. H. Chun, J. Phys. Chem. C, 2011, 115, 4491-4494.

18 T. Ma, H. Ariga, S. Takakusagi and K. Asakura, Thin Solid Films, 2018, 646, 12-16.

19 G. Q. Ding, Y. Zhu, S. M. Wang, Q. Gong, L. Sun, T. R. Wu, X. M. Xie and M. H. Jiang, Carbon, 2013, 53, 321-326.

20 Q. Chen, Y. J. Zhong, M. R. Huang, G. K. Zhao, Z. Zhen and H. W. Zhu, 2D Mater., 2018, 5, 035001.

21 G. B. Barina, Y. Song, I. F. Gimenez, A. G. S. Filho, L. S. Barreto and J. Kong, Carbon, 2015, 84, 82-90.

22 H. X. Ji, Y. F. Hao, Y. J. Ren, M. Charlton, W. H. Lee, Q. Z. Wu, H. F. Li, Y. W. Zhu, Y. P. Wu, R. Piner and R. S. Ruoff, $A C S$ Nano, 2011, 5, 7656-7661.

23 X. S. Li, W. W. Cai, J. H. An, S. Y. Kim, J. H. Nah, D. X. Yang, R. Piner, A. Velamakanni, I. Jung, E. Tutuc, S. K. Banerjee, L. Colombo and R. S. Ruoff, Science, 2009, 324, 1312-1314. 24 L. B. Gao, W. C. Ren, H. L. Xu, L. Jin, Z. X. Wang, T. Ma, L. P. Ma, Z. Y. Zhang, Q. Fu, L. M. Peng, X. H. Bao and H. M. Cheng, Nat. Commun., 2013, 3, 699.

25 R. R. Nair, P. Blake, A. N. Grigorenko, K. S. Novoselov, T. J. Booth, T. Stauber, N. M. R. Peres and A. K. Geim, Science, 2008, 320, 1308.

26 Y. F. Hao, M. S. Bharathi, L. Wang, Y. Y. Liu, H. Chen, S. Nie, X. H. Wang, H. Chou, C. Tan, B. Fallahazad, H. Ramanarayan, C. W. Magnuson, E. Tutuc, B. I. Yakobson, K. F. McCarty, Y. W. Zhang, P. Kim, J. Hone, L. Colombo and R. S. Ruoff, Science, 2013, 342, 720. 27 P. H. Ho, Y. C. Yeh, D. Y. Wang, S. S. Li, H. A. Chen, Y. H. Chung, C. C. Lin, W. H. Wang and C. W. Chen, ACS Nano, 2012, 6, 6215-6221.

28 F. H. L. Koppens, T. Mueller, P. Avouris, A. C. Ferrari, M. S. Vitiello and M. Polini, Nat. Nanotechnol., 2014, 9, 780-793.

29 A. D. Bartolomeo, G. Luongo, F. Giubileo, N. Funicello, G. Niu, T. Schroeder, M. Lisker and G. Lupina, 2D Mater., 2017, 4, 025075.

30 I. Goykhman, U. Sassi, B. Desiatov, N. Mazurski, S. Milana, D. Fazio, A. Eiden, J. Khurgin, J. Shappir, U. Levy and A. C. Ferrari, Nano Lett., 2016, 16, 3005-3013.

31 G. Wang, M. Zhang, D. Chen, Q. L. Guo, X. F. Feng, T. C. Niu, X. S. Liu, A. Li, J. W. Lai, D. Sun, Z. M. Liao, Y. Q. Wang, P. K. Chu, G. Q. Ding, X. M. Xie, Z. F. Di and X. Wang, Nat. Commun., 2018, 9, 5168. 